International Journal of Pure and Applied Mathematics

Volume 114 No. 1 2017, 1-15

ISSN: 1311-8080 (printed version); ISSN: 1314-3395 (on-line version)

url: http://www.ijpam.eu

doi: 10.12732/ijpam.v114i1.1

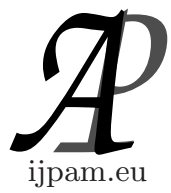

\title{
THE GENERALIZED PRE-OPEN COMPACT TOPOLOGY ON FUNCTION SPACES
}

\author{
S. Mishra ${ }^{1}$, S.M. Kang ${ }^{2} \S$, M. Kumar ${ }^{3}$ \\ ${ }^{1,3}$ Department of Mathematics \\ Lovely Professional University \\ Phagwara, Punjab, 144411, INDIA \\ ${ }^{2}$ Department of Mathematics and RINS \\ Gyeongsang National University \\ Jinju, 52828, KOREA
}

\begin{abstract}
The aim of this paper is to introduce new topology called generalized pre-open compact topology on the set of all real-valued continuous function on a Tychonoff space and compare this topology with other well-known topologies. Furthermore, we investigated relations of this topology with the induced mapping, as well as properties like metrizibility, submetrizibility and separability.
\end{abstract}

AMS Subject Classification: 54C10, 54C35, 54D65, 54E18, 54E35

Key Words: gpo-compact topology, induced mapping, metrizibility, submetrizibility and separability

\section{Introduction}

A function space is an interesting example of a topological space. In past, many researchers studies different kind of function spaces by placing different topologies on the set of functions. This study is help to understand relationship

\footnotetext{
Received: $\quad$ April 10, 2016

Revised: February 2, 2017

Published: $\quad$ April 17, 2017

(c) 2017 Academic Publications, Ltd. url: www.acadpubl.eu

${ }^{\S}$ Correspondence author
} 
between continuous function and a Baire measure on a Tychonoff space. The relationship between these two concepts can be clarified by the study of various locally convex topologies on spaces of continuous functions. The most interesting function space, $C(X)$, which is set of all real-valued continuous function on a Tychonoff space $X$, have been studied by defining three topologies like pointopen topology, compact-open topology and topology of uniform convergence. Among theses classical topologies, the compact-open topology introduced in 1945 by Fox [5]. Arens [3], and Arens and Dugundji [4] developed basic theory of compact-open topology. In 1952, Jackson [8] compared several topologies on function spaces and studied sequences of functions which converge uniformly on compact subset.

A classical result on relation between compact-open topology and topology of uniform convergence on $C(X)$ is that both topologies will be equal if and only if $X$ is compact. Since compactness is strong condition for this equality so there are many possibilities to find some topologies which lies between these two. Recently in this direction, there have been some topologies introduced like $\sigma$-compact open topology, the topology of uniform convergence on $\sigma$-compact, the topology of uniform convergence on bounded subsets, bounded-open topology, pseudocompact-open topology, $C$-compact-open topology and compact$G_{\delta}$-open topology. For more details we can see $[6,7,9,10,11,12,13,14,15,18]$.

It was found that compact spaces play an important role to define topologies on $C(X)$ and also help to investigate various properties function spaces. There are various weak forms of compact spaces was considered for defining above topologies. In this paper, our goal is to introduce and study another topology called generalized pre-open compact topology on $C(X)$ in term of generalized pre-open compact subset of $X$. We denote this topology by gpo and the corresponding space by $C_{g p o}(X)$.

The generalized compact space depend on generalized open set which was first time introduced by Levine [16]. The investigation on generalization of open set as well as closed set has led to significant contribution to the theory of generalization of continuity, separation axiom, covering properties and compactness. Many researchers have been done significant work in the direction of generalization of open set like semi-open, pre-open, $\alpha$-open, regular-open generalized pre-open etc. With the help of these generalized open sets several generalized form of compactness have been also introduced in last two decades which help to understand about various properties of topological spaces. In section 2 , we recalled some important definitions and results on generalized pre-open compact spaces. In sections 3, where we introduced generalized pre-open compact topology and compared with well know topologies on $C(X)$. In section 
4, we study the induced mapping on $C_{g p o}(X)$ and in last section, investigated metrizibility and separability of $C_{g p o}(X)$.

Throughout this paper we use following conventions. All spaces in this paper considered is a Tychonoff space that is, a complete regular Housdroff space. If $X$ and $Y$ are spaces with same underlying set, then $X=Y, X \leq Y$, $X<Y$ indicate that $X$ and $Y$ have same topology, that the topology $Y$ is finer or equal to the topology on $X$ and that the topology on $Y$ is strictly finer than the topology on $X$, respectively. The point-open topology, compactopen topology and uniform convergence topology on $C(X)$ is denoted by $p, k$ and $u$, respectively and corresponding spaces are denoted by $C_{p}(X), C_{k}(X)$ and $C_{u}(X)$, respectively. The $\mathbb{R}$ and $\mathbb{N}$ denote the space of real numbers and natural numbers respectively. The constant zero-function in $C(X)$ is denoted by 0 or sometimes $0_{X}$.

\section{Generalized Pre-Open Compact Spaces}

In this section, we recall some necessary definitions and results on generalized pre-open sets and generalized pre-open compact spaces.

Definition 2.1. [1] A subset $A$ of a space $X$ is a generalized pre-open set (briefly, gpo-set) if $\operatorname{cl}(A) \subseteq U$, whenever $U$ is a pre-closed subset such that $U \subseteq A$. Here, $\operatorname{cl}(A)$ denote closure of set $A$. Complements of gpo-sets are called a generalized pre-closed set (briefly, gpc-set).

The collection of all gpo-open set is denoted by $g p o(X)$.

With the help of gpo-set, Al-Hawary [2] defined a new compact space and discussed some properties of this space as follows.

Definition 2.2. A topological space $(X, \tau)$ is gpo-compact if every gpocover (a cover consisting of gpo-sets) of $X$ has a finite subcover.

Theorem 2.3. If $(X, \tau)$ is a gpo-compact space, then it is compact.

Theorem 2.4. If a space $(X, \tau)$ is gpo-irresolvable and gpo-compact, then it is strongly compact.

Theorem 2.5. If $(X, \tau)$ is a gpo-compact space, then it is s-closed.

Theorem 2.6. If a mapping $f: X \rightarrow Y$ is gpo-continuous surjective and $A$ is gpo-compact subset of $X$, then $f(A)$ is gpo-compact in $Y$. 


\section{Generalized Pre-Open Compact Topology on $C(X)$}

In this section we define three new topologies on $C(X)$ with the help of gpocompact subset of $X$.

First, consider a set for any gpo-compact subset $A$ of $X$ and any open subset $U$ of $\mathbb{R}$ as follows

$$
(A, U)=\{f \in C(X): f(A) \subseteq U\} .
$$

Suppose that the collection of all gpo-compact subset of $X$ is denoted by $g p o(X)$. The gpo-compact-open topology on $C(X)$ is generated by the subbase which is defined as

$$
\{(A, U): A \in g p o(X), U \text { is open in } \mathbb{R}\} .
$$

It can be easily verify that this subbasis actually generate the topology on $C(X)$, called gpo-compact-open topology on $C(X)$ and a new space corresponding to $C(X)$ is denoted by $C_{g p o}(X)$.

As we can verify that closure of any gpo-compact set is again gpo-compact and because $\overline{f(\bar{A})}=\overline{f(A)}$ for any $f \in C(X)$, so we can always consider closed gpo-compact subsets of $X$ in $(A, U)$.

Now we will define topology of uniform convergence on gpo-compact sets. For each $A \in \operatorname{gpo}(X)$ and $\epsilon>0$, let

$$
A_{\epsilon}=\{(f, g) \in C(X) \times C(X):|f(x)-g(x)|<\epsilon \text { for all } x \in A\} .
$$

It can be easily show that that the collection $\left\{A_{\epsilon}: A \in g p o(X), \epsilon>0\right\}$ is a base for some uniformity on $C(X)$. We denote a space corresponding to $C(X)$ with the topology induced by this uniformity by $C_{g p o, u}(X)$ and this topology is called the topology of uniform convergence on $\operatorname{gpo}(X)$.

For each $f \in C(X), A \in g p o(X)$, and $\epsilon>0$, let

$$
B_{A}(f, \epsilon)=\{g \in C(X):|f(x)-g(x)|<\epsilon \text { for all } x \in A\} .
$$

If $f \in C(X)$, the collection $\left\{B_{A}(f, \epsilon): A \in g p o(X), \epsilon>0\right\}$ forms a neighborhood base at $f$ in $C_{g p o, u}(X)$ and this collection forms a base for the topology of uniform convergence on $g p o(X)$. Here each set $B_{A}(f, \epsilon)$ is open in $C_{g p o, u}(X)$. If $g p o(X)$ covers $X$, then $C_{g p o, u}(X)$ is a Tychonoff space. If suppose $\operatorname{gpo}(X)=\{X\}$, then we get a topology of uniform convergence and it is denoted by $C_{u}(X)$. We can verify that for any $g p o(X), C_{u}(X) \leq C_{g p o, u}(X)$.

Now for each $A \in g p o(X)$ define $g p o$-semi norm $g o_{A}$ on $C(X)$ by

$$
\operatorname{gpo}_{A}(f)=\min \{1, \sup \{|f(x)|: x \in A\}\} .
$$


Also for each $A \in g p o(X)$ and $\epsilon>0$, let

$$
U_{A, \epsilon}=\left\{f \in C(X): \operatorname{gpo}_{A}(f)<\epsilon\right\} .
$$

Let $\mathcal{U}=\left\{U_{A, \epsilon}: A \in g p o(X), \epsilon>0\right\}$. It can be easily shown that for each $f \in C(X), f+\mathcal{U}=\{f+U: U \in \mathcal{U}\}$ forms a neighborhood base at $f$ We say that this topology is generated by the collection of gpo-seminorms $\left\{g o_{A}: A \in g p o(X)\right\}$.

Now we will establish a relation between above two defined topologies as $C_{g p o}(X)$ and $C_{g p o, u}(X)$.

Theorem 3.1. For any space $X$, the gpo-compact-open topology on $C(X)$ is same as the topology of uniform convergence on the gpo-compact subsets of $X$, that is, $C_{g p o}(X)=C_{g p o, u}(X)$.

Proof. Suppose that $(A, U)$ is subbasis open set in $C_{g p o}(X)$ and $f \in(A, U)$. Since $f(A)$ is compact, hence there exists $a_{1}, a_{2}, \ldots, a_{n}$ in $f(A)$ such that

$$
f(A) \subseteq \cup_{i=1}^{n}\left(a_{i}-\epsilon_{i}, a_{i}+\epsilon_{i}\right) \subseteq \cup_{i=1}^{n}\left(a_{i}-2 \epsilon_{i}, a_{i}+2 \epsilon_{i}\right) \subseteq U
$$

If we consider $g \in B_{A}(f, \epsilon)$ and $x \in A$, then this show that $|f(x)-g(x)|<\epsilon$ and there exist $i$ such that $\left|f(x)-a_{i}\right|<\epsilon_{i}$. Hence $\left|g(x)-a_{i}\right|<2 \epsilon_{i}$ and this show that $g(x) \subseteq U$ for all $x \in A$. So, $g(A) \subseteq U$, that is, $g \in(A, U)$. Hence we can say that $B_{A}(f, \epsilon) \subseteq(A, U)$. Let $\left.W=\cup_{i=1}^{n}\left(A_{i}, U_{i}\right]\right)$ be a basic neighborhood of $f$ in $C_{g p o}(X)$. Then there exists $\epsilon_{1}, \ldots, \epsilon_{i}$ such that

$$
f \in B_{A_{i}}\left(f, \epsilon_{i}\right) \subseteq\left(A_{i}, U_{i}\right) \cdot M
$$

Suppose that $A=\cup_{i=1}^{n} A_{i}$ and choose $\epsilon=\min _{1 \leq i \leq n}\left\{\epsilon_{i}\right\}$. Then $f \in$ $B_{A}(f, \epsilon) \subseteq W=\cap_{i=1}^{n}\left(A_{i}, U_{i}\right)=(A, U)$. Finally as we supposed $f \in(A, U)$ and we proved that $f \in B_{A}(f, \epsilon)$, we can say that $C_{g p o}(X) \leq C_{g p o, u}(X)$.

Now let $B_{A}(f, \epsilon)$ be a basic neighborhood of $f$ in $C_{g p o, u}(X)$. Since $f(A)$ is compact, there exist $a_{1}, a_{2}, \ldots, a_{n}$ in $f(A)$ such that $f(A) \subseteq \cup_{i=1}^{n}\left(a_{i}-\frac{\epsilon}{4}, a_{i}+\frac{\epsilon}{4}\right)$. Define $W_{i}=\left(a_{i}-\frac{\epsilon}{2}, a_{i}+\frac{\epsilon}{2}\right)$ and $A_{i}=c l_{A}\left(A \cap f^{-1}\left(a_{i}-\frac{\epsilon}{4}, a_{i}+\frac{\epsilon}{4}\right)\right)$. Here each $A_{i}$ will be gpo-compact and $A=\cup_{i=1}^{n}$. It is clear that $f \in \cap_{i=1}^{n}\left[A_{i}, W_{i}\right] \subseteq B_{A}(f, \epsilon)$. Let $g \in \cap_{i=1}^{n}\left[A_{i}, W_{i}\right]$ and $x \in A$. Then there exist $i$ such that $x \in A_{i}$ and $f(x) \in\left[\left(a_{i}-\frac{\epsilon}{4}, a_{i}+\frac{\epsilon}{4}\right]\right.$. Since $g(x) \in\left(a_{i}-\frac{\epsilon}{2}, a_{i}+\frac{\epsilon}{2}\right),|f(x)-g(x)|<\epsilon$. Hence $g \in B_{A}(f, \epsilon)$ and finally we can say that $C_{g p o, u}(X) \leq C_{g p o}(X)$.

Theorem 3.2. For any space $X$, the family $\{(A, U): A \in g p o(X)\}$, where $U$ is a bounded open interval in $\mathbb{R}$ forms a subbase for $C_{g p o}(X)$. 
Now we will compare gpo-compact-open topology with compact-open topology and topology of uniform convergence. As we know that if for any compact subset $A$ of $X$ and $f \in C(X)$ and $\epsilon>0$, the collection $\left\{B_{A}(f, \epsilon): A \in \mathcal{K}(X), \epsilon>\right.$ $0\}$ forms a neighborhood base at $f$ in the compact-open topology $k$ on $C(X)$. Where

$$
B_{A}(f, \epsilon)=\{g \in C(X):|f(x)-g(x)|<\epsilon, \forall x \in A\}
$$

and $\mathcal{K}(X)$ denote the collection of all compact subsets of $X$. The notion $C_{k}(X)$ denote the corresponding space of $C(X)$ when it is equipped with the compactopen topology $k$. Each element $B_{A}(f, \epsilon)$ is open in $C_{k}(X)$.

The collection $\left\{B_{X}(f, \epsilon): \epsilon>0\right\}$ forms a neighborhood base at each $f \in$ $C(X)$ and the topology generated by this base is called topology of uniform convergence $u$ on $C(X)$. The notation $C_{u}(X)$ denote the corresponding space when $C(X)$ equipped with the topology $u$.

Theorem 3.3. For any space $X$, gpo-compact-open topology is finer than compact-open topology that is $C_{k}(X)<C_{g p o}(X)$.

Proof. Let $\left(A_{k}, U\right)$ and $(A, U)$ be the subbasis elements for compact-open topology and gpo-compact-open topology respectively. Where $A_{k}$ and $A$ are compact and gpo-compact subsets of $X$ respectively. Let $f \in(A, U)$. Then by definition, $f(A)$ contained in open subset $U$ of $\mathbb{R}$. Now by Theorem 2.3 and as $f$ is continuous, $f(A)$ is compact in $U$. This show that $f \in\left(A_{k}, U\right)$. Therefore, $(A, U) \subset\left(A_{k}, U\right)$. Hence $C_{k}(X)<C_{g p o}(X)$.

For the space $X$, we can easily prove that topology of uniform convergence on $C(X)$ is finer than the gpo-compact-open topology that is $C_{g p o}(X)<C_{u}(X)$. Hence we can say that

Theorem 3.4. For any Tyhconoff space, $C_{k}(X) \leq C_{g p o}(X) \leq C_{u}(X)$.

Theorem 3.5. Every closed gpo-compact subset of $X$ is compact if and only if $C_{k}(X)=C_{g p o}(X)$.

Proof. For any subset $A$ of $X B_{\bar{A}}(f, \epsilon) \subseteq B_{A}(f, \epsilon)$. So, if every closed gpo-compact subset of $X$ is compact, then we can say that $C_{g p o}(X) \leq C_{k}(X)$. Hence in this case we can say that $C_{k}(X)=C_{g p o}(X)$.

Conversely, let $C_{k}(X)=C_{g p o}(X)$ and suppose that $A$ be any closed gpocompact subset of $X$. So set $B_{A}(0,1)$ as open lies in $C_{k}(X)$. This show that there exist a compact subset $K$ of $X$ and $\epsilon>0$ such that $B_{K}(0, \epsilon) \subseteq B_{A}(0,1)$. If possible, let $x \in A-K$. Then this implies that there exist a continuous mapping $g: A \rightarrow[0,1]$ such that $g(x)=1$ and $g(y)=0$ for all $y \in K$. Now 
here $g \in B_{K}(0, \epsilon)-B_{A}(0,1)$ which give a contradiction. Hence $A \subseteq K$ and consequently $A$ is compact.

Theorem 3.6. Every space $X$ is gpo-compact if and only if $C_{g p o}(X)=$ $C_{u}(X)$.

Proof. Let $X$ be a gpo-compact space. So, by definition it is possible for each $f \in C(X)$ and $\epsilon>0$ there exist a set $B_{X}(f, \epsilon)$ which is basic open in $C_{\text {gpo }}(X)$. Hence $C_{g p o}(X)=C_{u}(X)$.

Let us suppose $C_{g p o}(X)=C_{u}(X)$. Because a set $B_{X}(0,1)$ is a basic neighborhoods of a constant zero-mapping 0 in $C_{u}(X)$, then there exist a gpocompact subset $A$ of $X$ and $\epsilon>0$ such that $B_{A}(0, \epsilon) \subseteq B_{X}(0,1)$. With the help of complete regularity of $X$ it can be shown that $X=\bar{A}$. But the closure of a gpo-compact set is also gpo-compact. Hence $X$ is gpo-compact.

Theorem 3.7. Every closed countably compact subset of $X$ is compact if and only $C_{k}(X)=C_{g p o}(X)$, where $X$ is any normal Housdorff space.

Further we would like to find conditions when some spaces for which every closed gpo-compact subset is compact. Following definitions will help to find required conditions.

Definition 3.8. A space $X$ is said to be isocompact if every closed countably compact subset of $X$ is compact.

Definition 3.9. Similarly, a space $X$ is said to gpo-isocompact if every closed gpo-compact subsets of $X$ is compact.

With the help of above Definition 3.9, Theorem 3.7 also hold for gpoisocompact.

Theorem 3.10. Every space $X$ is gpo-isocompact if and only if $C_{g p o}(X)=$ $C_{k}(X)$.

Theorem 3.11. The space $X$ is isocompact if and only $C_{g p o}(X)=C_{k}(X)$, where $X$ is a normal space.

\section{Induced Mappings and gpo-Compact-Open Topology}

In this section, we discuss how the induced mapping related to gpo-compactopen topology. Following definitions and results will help to understand this relationship. 
Definition 4.1. Let $f: X \rightarrow Y$ be a continuous mapping between two topological spaces $X$ and $Y$. Then a mapping $f^{*}: C(Y) \rightarrow C(X)$ is said to be the induced mapping of $f$ if $f^{*}(g)=g \circ f$ for all $g$ in $C(Y)$.

Definition 4.2. Any mapping $f: X \rightarrow Y$ is said to be an almost onto mapping if $f(X)$ is dense in $Y$, where $X$ is any non-empty set and $Y$ is any topological space.

In this work, we will study nature of the induced mapping on $C(Y)$ to $C(X)$, when both are equipped with the gpo-compact-open topology.

Theorem 4.3. If $f: X \rightarrow Y$ be a continuous mapping between two topological spaces $X$ and $Y$, then the induced mapping of $f$ as $f^{*}: C_{g p o}(Y) \rightarrow$ $C_{g p o}(X)$ is continuous.

Proof. Let $g \in C_{g p o}(Y)$ and $B_{A}\left(f^{*}(g), \epsilon\right)$ be a basic neighborhood of $f^{*}(g)$ in $C_{\text {gpo }}(X)$. Then this show that $f^{*}\left(B_{A}(g, \epsilon)\right) \subseteq B_{A}\left(f^{*}(g), \epsilon\right)$ and consequently, $f^{*}$ is continuous.

Theorem 4.4. If $f: X \rightarrow Y$ be a continuous mapping between two topological spaces $X$ and $Y$, then the function $f^{*}: C(Y) \rightarrow C(X)$ is one-to-one if and only if $f$ is almost onto.

Proof. For sufficient: Let $g_{1}$ and $g_{2}$ in $C(Y)$ with $f^{*}\left(g_{1}\right)=f^{*}\left(g_{2}\right)$ and let $y$ in $f(X)$. Then for some $x \in X, y=f(x)$ and

$$
g_{1}(y)=g_{1}(f(x))=f^{*}\left(g_{1}\right)(x)=f^{*}\left(g_{2}\right)(x)=g_{2}(f(x))=g_{2}(y) .
$$

Since $f(X)$ is dense in $Y$, then $g_{1}=g_{2}$.

For necessary: Let there exists a $y$ in $Y-c l_{g p o}(f(X))$ and $p$ in $C[0,1]$ be a path in $\mathbb{R}$ so that $p(0) \neq p(1)$. Now the continuous function mapping $c l_{\text {gpo }}(f(X))$ onto $\{0\}$ and $y$ to 1 has an extension $\phi$ in $C(Y,[0,1])$. If $g=p \circ \phi$ and $c$ is the constant mapping taking $Y$ onto $\{p(0)\}$, then for each $x$ in $X$, $g(f(x))=p(0)=c(f(x))$. But then $f^{*}(g)=f^{*}(c)$, so that $f^{*}$ is not one-toone.

Theorem 4.5. If $f: X \rightarrow Y$ be a continuous mapping between two topological spaces $X$ and $Y$, then if $f^{*}: C(Y) \rightarrow C_{g p o}(X)$ is almost onto, then $f$ is one-to-one.

Proof. Suppose that $x_{1}$ and $x_{2}$ be two distinct elements of $X$. So, we have some $h$ in $C(X)$ such that $h\left(x_{1}\right) \neq h\left(x_{2}\right)$, and also there exist disjoint neighborhoods $U$ and $V$ of $h\left(x_{l}\right)$ and $h\left(x_{2}\right)$ in $\mathbb{R}$. Suppose that $S=\left[x_{l}, U\right] \cap$ 
$\left[x_{2}, V\right]$, which is a neighborhood of $h$ in $C_{g p o}(X)$. Then since $f^{*}$ is almost onto, there is some $g$ in $C(Y)$ with $f^{*}(g) \in S$. This show that $g\left(f\left(x_{l}\right)\right)$ and $g(f(x 2))$ in $U$ and $V$ respectively, so that $f\left(x_{1}\right) \neq f\left(x_{2}\right)$.

Following definition will helpful for discussion of embedding of $f^{*}$.

Definition 4.6. A continuous mapping $f: X \rightarrow Y$ is called gpo-covering if given any gpo-compact subset $A$ in $Y$, there exists a gpo-compact subset $C$ in $X$ such that $A \subseteq \operatorname{cl}(f(C))$.

Theorem 4.7. Suppose that $f: X \rightarrow Y$ be a continuous mapping between two spaces $X$ and $Y$. If $f^{*}: C_{g p o}(Y) \rightarrow C_{g p o}(X)$ is an embedding, then $f$ is a gpo-covering map.

Proof. Suppose that $A$ is gpo-compact subset of $Y$. Then $f^{*}\left(B_{A}\left(0_{Y}, 1\right)\right)$ is an open neighborhood of the zero function $)_{X}$ in $f^{*}\left(C_{g p o}(Y)\right)$. Now consider a gpo-compact subset $C$ of $X$ such that $0_{X} \in B_{C}\left(0_{X}, \epsilon\right) \cap f^{*}\left(C_{g p o}(Y)\right) \subseteq$ $f^{*}\left(B_{A}\left(0_{Y}, 1\right)\right.$. Now will proof that $A \subset c l(f(C))$. If it is possible, suppose $y$ in $A-\operatorname{cl}(f(C))$. This show that there exist a continuous function $g: Y \rightarrow[0,1]$ such that $g(y)=1$ and $g(c l(f(C)))=0$, by definition. Since $g(f(C))=0$ and

$$
f^{*}(g) \in B_{C}\left(0_{X}, \epsilon\right) \cap f^{*}\left(C_{g p o}(Y)\right) \subseteq f^{*}\left(B_{A}\left(0_{Y}, 1\right)\right) .
$$

Since $g^{*}$ is an injective mapping so $g \in B_{A}\left(0_{Y}, 1\right)$. But $y \in A$ show that $|g(y)|<1$. This is contradiction and hence $A \subseteq \operatorname{cl}(f(C))$. By definition, we can say that $f$ is gpo-covering.

The converse of above result not hold good but next theorem show that under some condition converse of above can establish.

Theorem 4.8. If a continuous mapping $f: X \rightarrow Y$ is gpo-covering, then $f^{*}: C_{g p o}(Y) \rightarrow C_{g p o}(X)$ is an embedding of $f$, where every gpo-compact subset of $Y$ is closed.

Proof. For each $a$ in $X$, there is possibility to find a gpo-compact subset $C$ of $X$ such that $\{a\} \subseteq f(C)$. So, $f$ is onto. Hence by Theorem 4.4, show that $f^{*}$ is one to one. Now our aim is to show that $f^{*}: C_{g p o}(Y) \rightarrow f^{*}\left(C_{g p o}(Y)\right)$ is an open map. Let $B_{A}(g, \epsilon)$ be a basic open set in $C_{g p o}(Y)$, where $A$ is any gpo-compact subset in $Y$ and $\epsilon>0$. Suppose that $h \in f^{*}\left(B_{A}(g, \epsilon)\right)$. Then there exist $h_{1} \in B_{A}(g, \epsilon)$ such that $f^{*}\left(h_{1}\right)=h$. Since $B_{A}(g, \epsilon)$ is open in $C_{g p o}(Y)$, then there exist a gpo-compact subset $D$ in $Y$, such that $B_{D}\left(h_{1}, \delta\right) \subseteq B_{A}(g, \epsilon)$, 
where $\delta>0$. As given that $f$ is gpo-covering so by definition, there exists a gpo-compact set $C$ in $X$ such that $D \subseteq f(C)$. Now we will show that

$$
B_{C}(h, \delta) \cap f^{*}\left(C_{g p o}(Y)\right) \subseteq f^{*} B_{D}\left(h_{1}, \delta\right)
$$

Let us choose $l \in C(Y)$ such that $f^{*}(l) \in B_{C}(h, \delta) \cap f^{*}\left(C_{g p o}(Y)\right)$. Since $D=f(C)$, for all $d \in D$, then there exist $c \in f(C)$ such that $d=f(c)$. Since $f^{*}(l) \in B_{C}(h, \delta)$ and

$$
\begin{aligned}
\left|l(d)-h_{1}(d)\right| & =\left|l(f(c))-h_{1}(f(c))\right| \\
& =\left|f^{*}(l)(c)-f^{*}\left(h_{1}\right)(c)\right| \\
& =\left|f^{*}(l)(c)-h(c)\right|<\delta .
\end{aligned}
$$

So we can say that $l \in B_{D}\left(h_{1}, \delta\right)$. This show that $f^{*}(l) \in f^{*}\left(B_{D}\left(h_{1}, \delta\right)\right)$. Hence

$$
B_{C}(h, \delta) \cap f^{*}\left(C_{g p o}(Y)\right) \subseteq f^{*}\left(B_{D}\left(h_{1}, \delta\right)\right) \subseteq f^{*}\left(B_{A},(g, \epsilon)\right)
$$

Consequently, $f^{*}\left(B_{A}(g, \epsilon)\right)$ is open in $f^{*}\left(C_{g p o}(Y)\right)$.

\section{Metrizibility and Separability of $C_{g p o}(X)$}

In this section, we will investigate two important properties as metrizibility and separability of $C_{g p o}(X)$. Following definitions will help in this investigation.

Definition 5.1. A space $(X, \tau)$ is said to be submetrizable if there is a topology $\tau^{*}$ that can be defined on $X$ such that $\left(X, \tau^{*}\right)$ is a metrizable space and $\tau^{*} \subset \tau$. In other words, $(X, \tau)$ is said to be submitriziable if there exist a continuous injective mapping $f:(X, \tau) \rightarrow(Y, d)$, where $(Y, d)$ is a metric space and $X$ is a completely regular Housdroff space.

Definition 5.2. The diagonal of a space $X$ is the subset of its square $X \times X$ that is defined by

$$
\Delta=\{(x, x): x \in X\}
$$

Definition 5.3. A $G_{\delta}$ is a set which can be written as a countable intersection of open set of a space $X$. If $\Delta$ is a $G_{\delta}$-set in $X \times X$, the space $X$ is said to have a $G_{\delta}$-diagonal. 
The properties of $G_{\delta}$ set are as: In metrizable spaces, every closed set is a $G_{\delta}$ set, the intersection of countably many $G_{\delta}$ sets is a $G_{\delta}$ set, and the union of finitely many $G_{\delta}$ sets is a $G_{\delta}$ set, a metric space has $G_{\delta}$-diagonal and all compact subsets, countably compact subsets and the singletons are $G_{\delta^{-}}$ sets in a submetrizable space. Every metrizable space has a zero-set diagonal consequently, every submetrizable space has also a zero-set-diagonal.

Definition 5.4. A space $X$ is called an $E_{0}$-space if every point in the space is a $G_{\delta}$-set. The submetrizable spaces are $E_{0}$-spaces.

Definition 5.5. A completely regular Hausdorff space $X$ is said to be $\sigma$-gpo-compact if there exists a sequence $\left\{A_{n}\right\}$ of gpo-compact sets in $X$ such that $X=\cup_{n=1}^{\infty} A_{n}$. A space $X$ is said to be almost $\sigma$-gpo-compact if it has a dense $\sigma$-gpo-compact subset.

Theorem 5.6. If $X$ is any space, then following are equivalent:

(1) $C_{g p o}(X)$ is submetriziable.

(2) Every gpo-compact subsets of $C_{g p o}(X)$ is a $G_{\delta}$ in $C_{g p o}(X)$.

(3) Every countable compact subsets of $C_{g p o}(X)$ is a $G_{\delta}$ in $C_{g p o}(X)$.

(4) Every compact subsets of $C_{g p o}(X)$ is a $G_{\delta}$ in $C_{g p o}(X)$.

(5) $C_{g p o}(X)$ is a $E_{0}$-space.

(6) $X$ is an almost $\sigma$-gpo-compact set.

(7) $C_{g p o}(X)$ has zero set-diagonal.

(8) $C_{g p o}(X)$ has $G_{\delta}$-diagonal.

Proof. By above definitions and properties we can show following implications:

$(1) \Rightarrow(2) \Rightarrow(3) \Rightarrow(4) \Rightarrow(5)$.

Here we are going to show that $(5) \Rightarrow(6)$.

As given $C_{g p o}(X)$ is an $E_{0}$-space so by definition any constant zero function 0 defined on $X$ will be $G_{\delta}$ set. Let $\{0\}=\cap_{n=1}^{\infty} B_{A_{n}}\left(0, \epsilon_{n}\right)$, where $A_{n} \in g p o(X)$ and $\epsilon_{n}>0$. Here we are going to show that $X=\operatorname{cl}\left(\cup_{i=1}^{\infty} A_{n}\right)$. Let $x_{0}$ be a arbitrary element in $X-l\left(\cup_{i=1}^{\infty} A_{n}\right)$. Then there exist a continuous mapping $f: X \rightarrow[0,1]$ such that $f(x)=0$, for all $x$ in $\cup_{i=1}^{\infty} A_{n}$ and by property of $X$ $f\left(x_{0}\right)=1$. Since $f(x)=0$ for all $x$ in $A_{n}$ and $f \in B_{A_{n}}\left(0, \epsilon_{n}\right)$ for all $n$ show that $f \in \cap_{i=1}^{\infty} B_{A_{n}}\left(0, \epsilon_{n}\right)=\{0\}$. So, $f(x)=0$ for all $x \in X$. But $f\left(x_{0}\right)=1$. This show a contradiction. Hence $X$ is almost $\sigma$-gpo-compact.

Theorem 5.7. Let $X$ be an almost $\sigma$-gpo-compact space and $\mathcal{P}$ is subset of $C_{\text {gpo }}(X)$. Then following are equivalent:

(1) $\mathcal{P}$ is compact.

(2) $\mathcal{P}$ is sequentially compact. 
(3) $\mathcal{P}$ is countably compact.

(4) $\mathcal{P}$ is gpo-compact.

Proof. It is easy to prove that $(2) \Rightarrow(3) \Rightarrow(4)$.

From the above Theorem $C_{g p o}(X)$ is submetriziable and so $\mathcal{P}$ will be also. A gpo-submetriziable space is metriziable hence it will be compact also. As we know that for a metriziable space all the above form of compactness are coincides. Hence we can say that $(1) \Rightarrow(2)$ and also $(4) \Rightarrow(1)$.

Now we are going to establish relations between some important topological properties and metrizibility for $C_{g p o}(X)$. But before this, first we define some important concepts like countable character, countable type, a $q$-space and a $M$-space which will help to understand these relations.

Definition 5.8. A subset $S$ of a space $X$ is said to have countable character if there is a sequence $\left\{W_{n}: n \in \mathbb{N}\right\}$ of open subsets in $X$ such that $S \subseteq W_{n}$ for each $n$, and if $W$ is any open set containing $S$, then $W_{n} \subseteq W$ for some $n$.

Definition 5.9. A space $X$ is said to be of (pointwise) countable type if each (point) compact set is contained in a compact set having countable character.

Definition 5.10. A topological space $X$ is said to be a $q$-space if for every $x \in X$ has a sequence $\left\{U_{i}\right\}$ of neighborhoods satisfying the condition: If $\left\{x_{i}\right\}$ is an infinite sequence of points in $X$ such that $x_{i} \in U_{i}$ for each $i$, then $\left\{x_{i}\right\}$ has an accumulation point in $X$.

Definition 5.11. A topological space is said to be a $M$-space if it can be mapped onto a metric space by a quasi-perfect mapping (a continuous closed mapping in which inverse images of points are countably compact). This space is stronger than a $q$-space.

As we know that a topological space is said to be a hemi-compact space if it has a sequence of compact subsets such that every compact subset of the space lies inside some compact set in the sequence. This concept is used to characterize the metrizability of $C_{k}(X)$. Now for discussion of metrizibility of $C_{g p o}(X)$, first we define a hemi-gpo-compact space as follows.

Definition 5.12. A topological space $X$ is said to be a hemi-gpo-compact space if there exists a sequence of gpo-compact set $\left\{A_{n}\right\}$ in $X$ such that for any gpo-compact subset $A$ of $X, A \subseteq A_{n}$ holds for some $n \in \mathbb{N}$.

Now we are in position to relate the metriczibility of $C_{g p o}(X)$ with properties discussed above. 
Theorem 5.13. For any space $X$ following are equivalent:

(1) $C_{g p o}(X)$ is metrizable.

(2) $C_{g p o}(X)$ is of first countable.

(3) $C_{g p o}(X)$ is of countable type.

(4) $C_{\text {gpo }}(X)$ is of pointwise countable type.

(5) $C_{\text {gpo }}(X)$ has a dense subspace of pointwise countable type.

(6) $C_{g p o}(X)$ is a $M$-space.

(7) $C_{\text {gpo }}(X)$ is a $q$-space.

(8) $X$ is a hemi-gpo-compact space.

Proof. From the above discussion, we can directly show that $(1) \Rightarrow(3) \Rightarrow$ $(4) \Rightarrow(7),(1) \Rightarrow(6) \Rightarrow(7)$ and also $(1) \Rightarrow(2) \Rightarrow(7)$.

We will prove $(4) \Leftrightarrow(5)$.

As we know that if $D$ is any dense subset of a space $X$ and $A$ is a compact subset of $D$, then $A$ has countable character in $D$ if and only if $A$ is of countable character in $X$. Since $C_{g p o}(X)$ is a locally convex space, it is homogenous. So, with the help of this result and above discussion we can say that $(4) \Leftrightarrow(5)$.

Next, we will prove $(7) \Rightarrow(8)$. Let $C_{g p o}(X)$ be a $q$-space So, there exist a sequence $\left\{V_{n}: n \in \mathbb{N}\right\}$ of neighborhoods of the zero-function 0 in $C_{g p o}(X)$ such that $f_{n} \in V_{n}$ for each $n$, then the set $\left\{f_{n}: n \in \mathbb{N}\right\}$ has a cluster point in $C_{\text {gpo }}(X)$. Now for each $n$, there exists a closed gpo-compact subset $A_{n}$ of $X$ and $\epsilon_{n}>0$ such that $0 \in B_{A_{n}}\left(0, \epsilon_{n}\right)$.

Let $A$ be gpo-compact subset of $X$. Suppose that $A$ is not subset of $A_{n}$ for any $\mathbb{N}$. Then for each $n \in \mathbb{N}$, there exists $a_{n} \in A-A_{n}$. Hence for each $n \in \mathbb{N}$ there exists a continuous function $f_{n}: X \rightarrow[0,1]$ such that $f_{n}\left(a_{n}\right)=n$ and $f_{n}(x)=0$ for all $x \in A_{n}$. Hence it is clear that $f_{n} \in B_{A_{n}}\left(0, \epsilon_{n}\right)$. But the sequence $\left\{f_{n}\right\}_{n \in \mathbb{N}}$ does not have a cluster point in $C_{g p o}(X)$. If possible, let that this sequence has a cluster point $f \in C_{g p o}(X)$. Then for each $k \in \mathbb{N}$, there exists a positive integer $n_{k}>k$ such that $f_{n_{k}} \in B_{A}(f, 1)$. So, for all $k \in \mathbb{N}, f\left(a_{n_{k}}\right)>f_{n_{k}}\left(a_{n_{k}}-1\right)=n_{k}-1 \geq k$. This show that $f$ is unbounded on the gpo-compact set $A$. Hence the sequence $f_{n}$ cannot have a cluster point in $C_{g p o}(X)$ and consequently, $C_{g p o}(X)$ fails to be a $q$-space. Hence, $X$ must be a hemi-gpo-compact space.

Finally, we will show that $(8) \Rightarrow(1)$. As we know that if the topology of a locally convex Hausdroff space is generated by a countable family of seminorms, then it is metrizable. Now the locally convex topology on $C(X)$ generated by the countable family of seminorms $\left\{p_{A_{n}}: n \in \mathbb{N}\right\}$ is metrizable and weaker than the gpo-compact-open topology. However, since for each gpo-compact set $A$ in $X$, there exists $A_{n}$ such that $A \subseteq A_{n}$, the locally convex topology generated 
by the family of seminorms $\left\{p_{A}: A \in g p o(X)\right\}$, that is the gpo-compact-open topology, is weaker than the topology generated by the family of seminorms $\left\{p_{A}: n \in \mathbb{N}\right\}$. Hence, $C_{\text {gpo }}(X)$ is metrizable.

At the end of this paper we will investigate about separability of $C_{g p o}(X)$. Following results show the relation between separability of $C_{g p o}(X)$ with separability of other spaces like $C_{p}(X)$ and $C_{k}(X)$.

Theorem 5.14. For any space $X$, the following are equivalent:

(1) $C_{\text {gpo }}(X)$ is separable.

(2) $C_{p}(X)$ is separable, where $p$ is point-open topology.

(3) $C_{k}(X)$ is separable.

(4) $X$ has a weaker separable metriziable topology.

Proof. From the Corollary 4.2.2 in [17] that (2), (3) and (4) are equivalent. Also, since $C_{p} \subseteq C_{g p o}(X)$, so (1) $\Rightarrow(2)$.

We will prove $(4) \Rightarrow(1)$. As we know that if $X$ has a weaker separable metrizable topology, then $X$ is realcompact. Hence, $X$ is gpo-isocompact. Consequently, $C_{\text {gpo }}(X)=C_{k}(X)$. Since $(4) \Rightarrow(3), C_{g p o}(X)$ is separable.

\section{References}

[1] T. Al-Hawary, Generalized preopen sets, Questions Answers, Gen. Topology, 29 (2011), 73-80.

[2] T. Al-Hawary, On generalized preopen sets, Proyecciones 32 (2013), 47-60, doi: 10.4067/S0716-09172013000100004.

[3] R. Arens, A topology for spaces of transformations, Ann. of Math., 47 (1946), 480-495, doi: $10.2307 / 1969087$.

[4] R. Arens, J. Dugundji, Topologies for function spaces, Pacific J. Math., 1 (1951), 5-31.

[5] R.H. Fox, On topologies for function spaces, Bull. Amer. Math Soc., 51 (1945), 429-432, doi: 10.1090/S0002-9904-1945-08370-0.

[6] P. Garg, S. Kundu, The compact- $G_{\delta}$-open topology on $C(x)$, Topology Appl., 159 (2012), 2082-2089, doi: 10.1016/j.topol.2012.01.017.

[7] D. Gulick, The $\sigma$-compact-open topology and its relatives, Math. Scand., 30 (1972), 159-176, doi: 10.7146/math.scand.a-11072.

[8] J.R. Jackson, Comparison of topologies on function spaces, Proc. Amer. Math. Soc., 3 (1952), 156-158, doi: 10.1090/S0002-9939-1952-0046031-5.

[9] S. Kundu, Spaces of continuous linear functional: something old and something new, Topology Proc., 14 (1989), 113-129.

[10] S. Kundu, The metrizability and completeness of the support-open topology on $C(x)$, Topology Appl., 157 (2010), 1119-1126, doi: 10.1016/j.topol.2010.01.002. 
[11] S. Kundu, P. Garg, The pseudocompact-open topology on $C(x)$, Topology Proc., 30 (2006), 279-299.

[12] S. Kundu, P. Garg, Countability properties of the pseudocompact-open topology on $C(x)$ : a comparative study, Rend. Istit. Mat. Univ. Trieste, 39 (2007), 421-444.

[13] S. Kundu, P. Garg, The compact-open topology: a new perspective, Topology Appl., 156 (2009), 686-696, doi: 10.1016/j.topol.2008.09.006.

[14] S. Kundu, R.A. McCoy, Topologies between compact and uniform convergence on function spaces, Internat. J. Math. Math. Sci., 16 (1993), 101-110, doi: $10.1155 /$ S0161171293000122.

[15] S. Kundu, A.B. Raha, The bounded-open topology and its relatives, Rend. Istit. Mat. Univ. Trieste, 27 (1995), 61-77.

[16] N. Levine, Generalized closed sets in topology, Rend. Circ. Mat. Palermo, 2 (1970), 89-96, doi: 10.1007/BF02843888.

[17] R.A. McCoy, I. Ntantu, Topological Properties of Spaces of Continuous Functions, Lecture notes in mathematics, 1315, Springer-Verlag, Berlin, 1988.

[18] A.V. Osipov, The $C$-compact-open topology on function spaces, Topology Appl., 159 (2012), 3059-3066, doi: 10.1016/j.topol.2012.05.018. 
